\title{
Green synthesis of a typical chiral stationary phase of cellulose-tris(3, 5-dimethylphenylcarbamate)
}

\author{
Run-Qiang Liu ${ }^{1,2}$, Lian-yang Bai ${ }^{1,3^{*}}$, Yi-Jun Zhang ${ }^{2}$ and Yu-Ping Zhang ${ }^{2^{*}}$
}

\begin{abstract}
Background: At present, the study on the homogeneous-phase derivatization of cellulose in ionic liquid is mainly focused on its acetylation. To the best of our knowledge, there has been no such report on the preparation of cellulose-tris(3,5-dimethylphenylcarbamate) (CDMPC) with ionic liquid 1-allyl-3-methyl-imidazolium chloride (AmimCl) so far.

Results: With ionic liquid 1-allyl-3-methylimidazolium chloride (AmimCl) as a reaction solvent, cellulose-tris(3,5dimethylphenylcarbamate) (CDMPC) was synthesized by the reaction of 3,5-dimethylphenyl isocyanate and soluble microcrystalline cellulose in a homogeneous phase. The synthesized CDMPC was then coated onto the surfaces of aminopropyl silica gel to prepare a chiral stationary phase (CSP). The prepared CSP was successfully used in chiral separation of seven racemic pesticides by high performance liquid chromatography (HPLC). Good chiral separation was obtained using $n$-hexane and different modifiers as the mobile phases under the optimal percentage and column temperature, with the resolution of metalaxyl, diniconazole, flutriafol, paclobutrazol, hexaconazole, myclobutanil and hexythiazox of 1.73, 1.56, 1.26, 1.00, 1.18, 1.14 and 1.51, respectively. The experimental results suggested it was a good choice using a green solvent of AmimCl for cellulose functionalization.
\end{abstract}

Conclusion: CDMPC was successfully synthesized as the chiral selector by reacting 3, 5-dimethylphenyl isocyanate with dissolved microcrystalline cellulose in a green ionic liquid of AmimCl.

Keywords: Ionic liquid, AmimCl, HPLC, Chiral stationary phase, Pesticides, Chiral separation

\section{Findings}

Enantioseparation is an effective approach in the determination of purity of enantiomers and preparation of a single enantiomer in the research fields, such as chiral drugs, natural products, and agricultural chemicals [1]. The key in the chiral technique is the design and application of chiral stationary phases (CSPs) [2-5]. At present, the polysaccharide derivatives, especially benzoate and phenylcarbamate derivatives of cellulose and starch, are most popular CSPs for direct separation of enantiomers with HPLC due to their strong chiral recognition capability [6-9]. Generally, the synthesis of cellulose benzoates started from cellulose suspension in pyridine and carried out heterogeneously. Ionic liquid is an organic salt in the

\footnotetext{
*Correspondence: blyang2006@163.com; beijing2008zyp@163.com

${ }^{1}$ College of Plant Protection, Hunan Agricultural University, Changsha 410128, P.R. China

${ }^{2}$ Henan Institute of Science and Technology, Xinxiang 453003, P.R. China

Full list of author information is available at the end of the article
}

form of liquid under normal temperature, which has attracted wide attention from the chemistry community for its advantages of low melting point, non-volatility, and adjustable structure and property, etc. [10-12]. Recently, Swatloski et al. found that 1-butyl-3-methylimidazolium chloride $(\mathrm{BmimCl})$ ionic liquid was able to dissolve cellulose [13], which opened a new field for the study and development of cellulose solvent system. Wu et al., not only proved 1-allyl-3-methylimidazolium chloride (AmimCl) to be a good solvent for cellulose, but also successfully synthesized cellulose acetate in it [14]. At present, the study on the homogeneous-phase derivatization of cellulose in an ionic liquid is mainly focused on its acetylation. There has been no report on the esterification of cellulose in an ionic liquid of $\mathrm{AmimCl}$ so far.

In this study, CDMPC, as a chiral selector, was firstly prepared in an ionic liquid of $\mathrm{AmimCl}$, followed by the characterization of FTIR, ${ }^{1} \mathrm{HNMR}$ and elementary analysis. After it was coated on the surfaces of silica gel and
(C) Chemistry Central

(c) 2013 Liu et al.; licensee Chemistry Central Ltd. This is an Open Access article distributed under the terms of the Creative Commons Attribution License (http://creativecommons.org/licenses/by/2.0), which permits unrestricted use, distribution, and reproduction in any medium, provided the original work is properly cited. 
packed into a chromatographic steel column, seven racemic pesticides including diniconazole, flutriafol, paclobutrazol, myclobutanil, hexaconazole, metalaxyl and hexythiazox, were selected for the chromatographic evaluation of enantioselective ability in normal- phase HPLC.

\section{Experiment}

\section{Equipments and reagents}

Chromatographic measurements were made on a Agilent 1100 HPLC system (Agilent Technologies, Inc., Walbronn, Germany) equipped with a quaternary pump, a vacuum degasser module, a Rheodyne $7725 \mathrm{i}$ injector with a $20 \mu \mathrm{L}$ sample loop, a temperature controlled column compartment and a variable wavelength UV detector. FTIR spectra were recorded in the range 400-4000 $\mathrm{cm}^{-1}$ with $4 \mathrm{~cm}^{-1}$ resolution with a BRUKER TENSOR27 system (Bruker Scientific Technology Co. Ltd., Karlsruhe, Germany). The $400 \mathrm{MHz} 1 \mathrm{H}$ NMR spectra were measured on a BRUKER ARX 400 spectrometers (Bruker Scientific Technology Co. Ltd., Karlsruhe, Germany).

AmimCl was purchased from Shanghai Cheng Jie Chemical Co. Ltd. (Shanghai, China). $5 \mu \mathrm{m}$ spherical silica gel (Kromasil, pore size $10 \mathrm{~nm}$ ) was purchased from Akzo Noble N.V. (Nacka, Sweden). Microcrystalline cellulose (Avicel, DP = 200), tetrahydrofuran (THF), 3-aminopropyltriethoxysilane and 3, 5-dimethylphenyl isocyanate were purchased from Aladdin Chemistry Co. Ltd. (Shanghai, China). Diniconazole (97.4\%), flutriafol (97\%), paclobutrazol (97.4\%) and myclobutanil (98\%) were provided by Hunan Research Institute of Chemical Industry, hexaconazole (95\%), metalaxyl (98\%) and hexythiazox (98\%)were provided by Hunan Institute for Food and Drug Control.

\section{Synthesis of CDMPC in AmimCI}

Synthesis of CDMPC was carried out according to the previous report [15], but the great difference was that an ionic liquid of AmimCl was used instead of traditional pyridine solvent.

As shown in Figure 1, $1.00 \mathrm{~g}$ of dry microcrystalline cellulose was transferred into a $100 \mathrm{ml}$ three-neck flask, and the flask was added with $34.00 \mathrm{~g}$ of AmimCl (1).
The solution was stirred magnetically for dissolution $\left(90^{\circ} \mathrm{C}, 3 \mathrm{~h}\right)$. The flask was added with $10.00 \mathrm{~mL}$ of $3,5-$ dimethylphenyl isocyanate (2), then the solution was refluxed for $48 \mathrm{~h}$. The whole process was conducted under the protection of $\mathrm{N}_{2}$. After the solution was cooled down, it was added with $800 \mathrm{ml}$ of methanol. The sediment was washed with $200 \mathrm{ml}$ of methanol, and then dried for $12 \mathrm{~h}$ under vacuum at $80^{\circ} \mathrm{C} .2 .93 \mathrm{~g}$ of white solid of CDMPC was produced with a yield of $79.19 \%$.

\section{Preparation of CDMPC-coated CSP}

CDMPC-coated CSP was prepared according to the published reference [16]. Firstly, $3.00 \mathrm{~g}$ of silica gel was transferred into toluene, and sufficiently reacted with 3aminopropyltriethoxysilane at $110^{\circ} \mathrm{C}$ for $48 \mathrm{~h}$ to obtain aminopropyl-silica gel (APS). Secondly, $0.45 \mathrm{~g}$ of the synthesized CDMPC was weighed and dissolved in $30 \mathrm{~mL}$ of tetrahydrofuran. Then the solution was added to $2.55 \mathrm{~g}$ of APS, and dried for $8 \mathrm{~h}\left(60^{\circ} \mathrm{C}\right.$, vacuum condition) after the solvent was removed under vacuum at room temperature. A CDMPC-coated CSP with coating quantity of $15 \%$ was thus prepared.

\section{Column packing}

With isopropanol as slurry solvent and the mixture of nhexane/isopropanol (90/10, v/v) as displacement fluid, the CDMPC-coated CSP was filled into the stainless-steel column $(250 \mathrm{~mm} \times 4.6 \mathrm{~mm})$ under a pressure of $7000 \mathrm{psi}$.

\section{Results and discussion}

\section{Characterization of CDMPC}

FTIR spectra of microcrystalline cellulose and CDMPC were compared in Figure 2. The spectrum of CDMPC had a significantly weaker - $\mathrm{OH}$ absorption peak at $3415.31 \mathrm{~cm}^{-1}$, suggesting that the $-\mathrm{OH}$ groups of cellulose were mostly replaced. Peak at $2902.34 \mathrm{~cm}^{-1}$ stands for the stretching vibration peak of $-\mathrm{CH}_{3}$ group, the absorption peaks of the benzene ring appeared at $1616.06 \mathrm{~cm}^{-1}$, $1544.70 \mathrm{~cm}^{-1}$ and $1455.99 \mathrm{~cm}^{-1}$, the absorption peak of the carbonyl group was found at $1737.55 \mathrm{~cm}^{-1}$. These all suggest that the $-\mathrm{OH}$ groups on microcrystalline cellulose have reacted with 3,5-dimethylphenyl isocyanate.

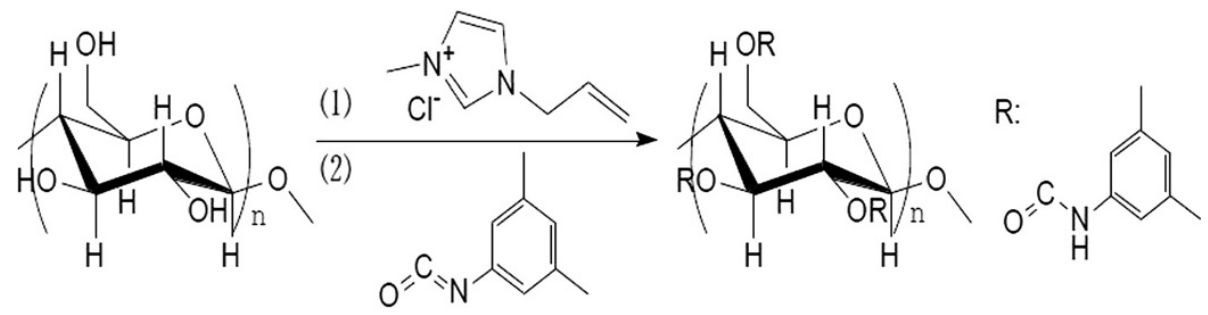

Figure 1 Synthesis of CDMPC in AmimCl. 


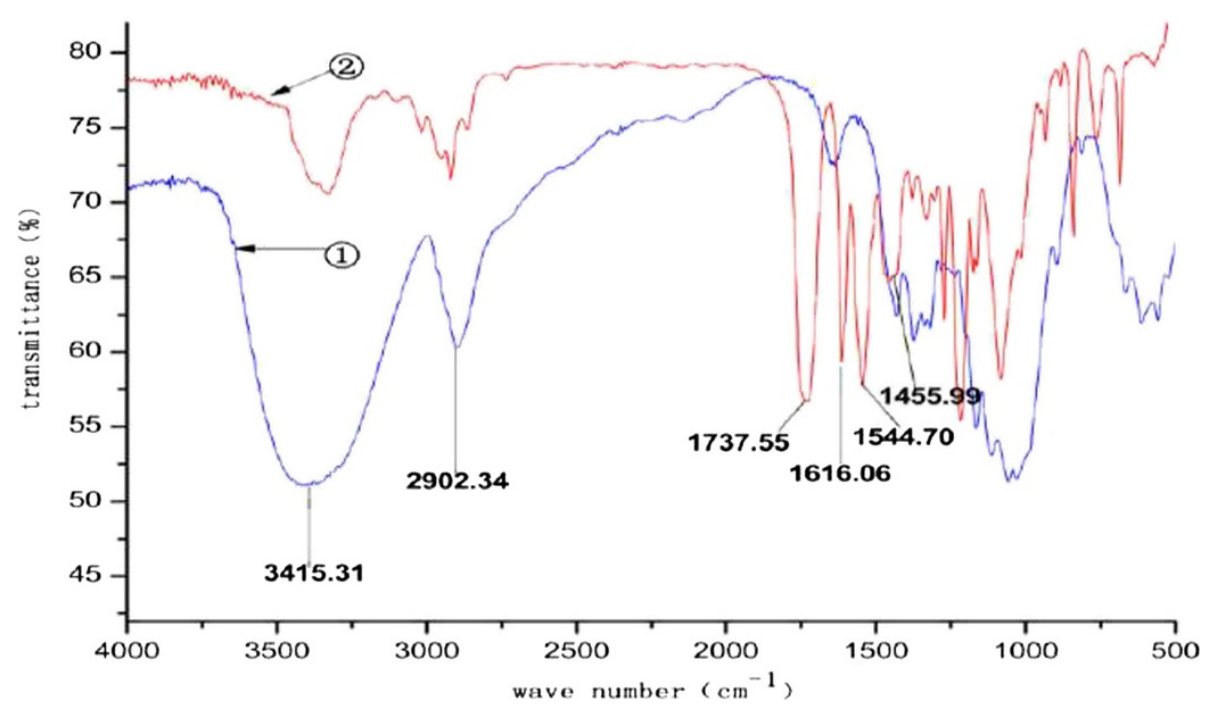

Figure 2 FTIR spectra of cellulose and CDMPC. (1) Cellulose, (2) CDMPC.

The obtained CDMPC was also determined by the ${ }^{1} \mathrm{H}$ NMR $\left(400 \mathrm{MHz}, 50^{\circ} \mathrm{C}\right.$, DMSO- $\left.\mathrm{d}_{6}\right)$. As shown in Figure 3, the chemical shifts $(\delta, \mathrm{ppm})$ could be observed at different positions of 8.5-8.9 (N-H), 6.3-7.1 (Ar-H), 3.5-5.1 (Glucose-H), 1.8-2.2 (Ar- $\left.\mathrm{CH}_{3}\right)$.

From the molecular formula of CDMPC $\left(\mathrm{C}_{33} \mathrm{H}_{37} \mathrm{O}_{8} \mathrm{~N}_{3}\right)$, its theoretical components of each element are $\mathrm{C} 65.70 \%$, $\mathrm{H} 6.18 \%$ and N 6.96\%, respectively. In contrast, the measured components of CDMPC by elemental analysis are $\mathrm{C}$ $64.40 \%, \mathrm{H} 6.26 \%$ and $\mathrm{N} 6.73 \%$. The comparable results showed no obvious difference of element content was found, suggesting that CDMPC was successfully synthesized by the developed method.

\section{Chromatographic evaluation for the coated CSPS}

Diniconazole, flutriafol, paclobutrazol and hexaconazole all contain benzene rings and hydroxyls in their molecular structures, hexythiazox and metalaxyl contain benzene rings and carbonyls in their molecular structures, myclobutanil, diniconazole, paclobutrazol and hexaconazole contain chlorphenyls and 1, 2, 4-triazolyls in their molecules. The above groups may have dipole-dipole interaction, hydrogen bond interaction and $\pi-\pi$ interaction with the stationary phases. Generally, the polarity of mobile phase can be changed by changing the alcohol ratio in mobile phase, which in turn affects the retention time and resolution in enantioseparation [17]. In order to

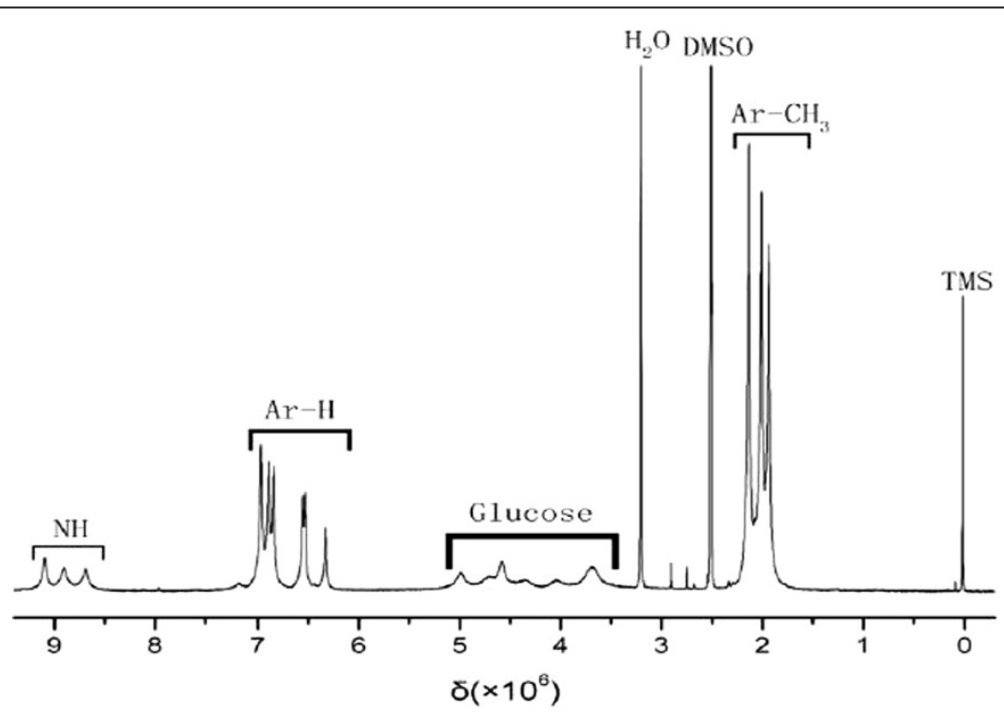

Figure 3 NMR spectrum of CDMPC. 
Table 1 The resolutions under optimized conditions

\begin{tabular}{|c|c|c|c|c|c|}
\hline Sample & Optimized condition & $k 1$ & $k 2$ & a & Rs \\
\hline Paclobutrazol & $25^{\circ} \mathrm{C}, 2 \%$ isopropanol in $n$-hexane, $1.0 \mathrm{~mL} \cdot \mathrm{min}^{-1}, 222 \mathrm{~nm}$ & 9.93 & 11.52 & 1.16 & 1.00 \\
\hline Hexaconazole & $25^{\circ} \mathrm{C}, 2 \%$ isopropanol in $n$-hexane, $1.0 \mathrm{~mL} \cdot \mathrm{min}^{-1}, 230 \mathrm{~nm}$ & 8.81 & 10.48 & 1.19 & 1.18 \\
\hline Hexythiazox & $25^{\circ} \mathrm{C}, 2 \%$ isopropanol in $n$-hexane, $1.0 \mathrm{~mL} \cdot \mathrm{min}^{-1}, 230 \mathrm{~nm}$ & 3.10 & 3.95 & 1.27 & 1.51 \\
\hline Diniconazole & $25^{\circ} \mathrm{C}, 20 \%$ isopropanol in $n$-hexane, $1.0 \mathrm{~mL} \cdot \mathrm{min}^{-1}, 230 \mathrm{~nm}$ & 0.60 & 0.91 & 1.52 & 1.56 \\
\hline Flutriafol & $5^{\circ} \mathrm{C}, 5 \%$ isopropanol in $n$-hexane, $1.0 \mathrm{~mL} \cdot \mathrm{min}^{-1}, 214 \mathrm{~nm}$ & 15.16 & 19.11 & 1.26 & 1.26 \\
\hline Myclobutanil & $25^{\circ} \mathrm{C}, 15 \%$ isopropanol in $n$-hexane, $1.0 \mathrm{~mL} \cdot \mathrm{min}^{-1}, 210 \mathrm{~nm}$ & 7.48 & 8.98 & 1.20 & 1.14 \\
\hline Metalaxyl & $25^{\circ} \mathrm{C}, 5 \%$ ethanol in $n$-hexane, $1.0 \mathrm{~mL} \cdot \mathrm{min}^{-1}, 214 \mathrm{~nm}$ & 4.75 & 6.11 & 1.29 & 1.73 \\
\hline
\end{tabular}

preliminarily study the separation effects of seven racemic pesticides and investigate the impact of polarity on separation, we selected isopropanol as the polar modifier. The impact of modifier (isopropanol/ethanol) and separation temperature on the retention time and stereo selectivity of enantiomer separation of 7 racemic pesticides on the self-made CDMPC chiral column were investigated in detail. When n-hexane/isopropanol (98/ $2, \mathrm{v} / \mathrm{v}$ ) were used as the mobile phases with a flow rate of $1.0 \mathrm{ml} / \mathrm{min}$ at $25^{\circ} \mathrm{C}$, the resolution of paclobutrazol, hexaconazole and hexythiazox was 1.00, 1.18 and 1.51, respectively, the resolution of diniconazole was 1.56 when $n$-hexane/isopropanol $(80 / 20, v / v)$ were used as the mobile phases at $25^{\circ} \mathrm{C}$, the resolution of flutriafol was 1.26 when $n$-hexane/isopropanol $(95 / 5, v / v)$ were used as the mobile phases at $20^{\circ} \mathrm{C}$, the resolution of myclobutanil was 1.14 when $n$-hexane/isopropanol (85/ $15, \mathrm{v} / \mathrm{v}$ ) were used as the mobile phases at $25^{\circ} \mathrm{C}$, the resolution of metalaxyl was 1.73 when $n$-hexane/ethanol $(95 / 5, \mathrm{v} / \mathrm{v})$ were used as the mobile phases at $25^{\circ} \mathrm{C}$. The optimal separation chromatograms of 7 racemic pesticides were shown in Table 1 and Figure 4.

The direct enantioseparation of seven pesticides has been achieved, demonstrating the high chiral recognition abilities for the coated CDMPC in chiral HPLC. Good chiral separation was probably attributed to the hydrogen bond interaction, dipole-dipole interaction and $\pi-\pi$ interaction between the CSPs and the recognized analyte [18]. a



d

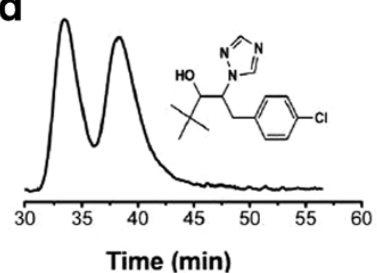

g

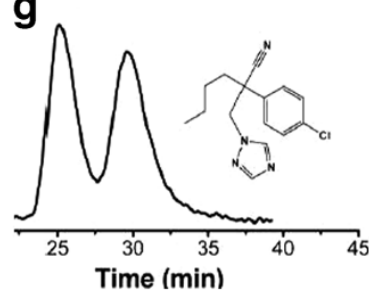

b

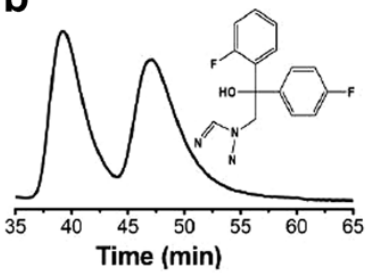

e

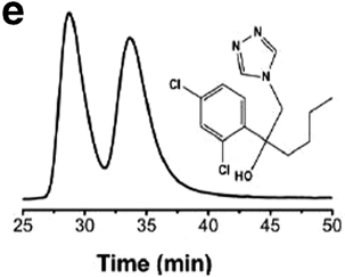

C
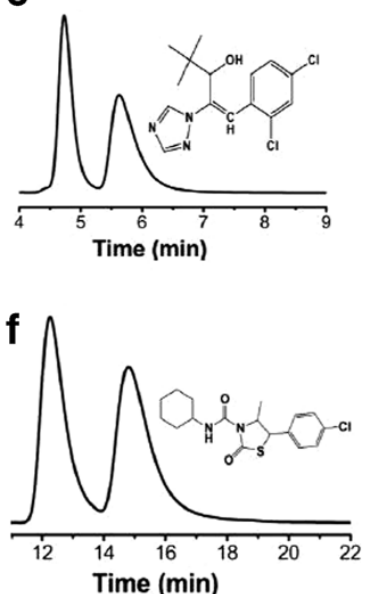


\section{Conclusions}

With the green solvent of $\mathrm{AmimCl}$ as a homogenous reaction medium, CDMPC was successfully prepared by the reaction of 3, 5-dimethylphenyl isocyanate with soluble microcrystalline cellulose. Its chemical structure was further proved by the FTIR, ${ }^{1} \mathrm{HNMR}$ and elementary analysis. The prepared CDMPC-coated CSPs was successfully used in chiral separation of seven pesticides by HPLC. Seven racemic pesticides were well separated under the optimized chromatographic conditions. It suggested that it was a good alternative to synthesize the selector in the presence of a low-cost, environmentally friendly cellulose solvent.

\section{Description of additional material}

The synthetic method of CDMPC by reacting $3,5-$ dimethylphenyl isocyanate with dissolved microcrystalline cellulose in a green ionic liquid of AmimCl are available. The data characterization of FTIR, 1HNMR and elemental analysis are also available.

\section{Abbreviations}

CDMPC: Cellulose-tris(3,5-dimethylphenylcarbamate); AmimCl: 1-allyl-3methyl-imidazolium chloride; CSPs: Chiral stationary phase; HPLC: High performance liquid chromatography; THF: Tetrahydrofuran;

APS: Aminopropyl-silica gel.

\section{Competing interests}

The authors declare that they have no competing interests.

\section{Authors' contributions}

BLY and ZYP initiated and designed the study. All authors contributed to data analyses and to finalizing the manuscript. All authors have read and approved the final version.

\section{Acknowledgements}

The financial supports by the National Natural Science Foundation of China (Nos. 30070506 and 30771436) are gratefully acknowledged.

\section{Author details}

${ }^{1}$ College of Plant Protection, Hunan Agricultural University, Changsha 410128, P.R. China. ${ }^{2}$ Henan Institute of Science and Technology, Xinxiang 453003, P.R. China. ${ }^{3}$ Hunan Academy of Agricultural Sciences, Changsha 410125, P.R. China.

Received: 2 April 2013 Accepted: 11 June 2013

Published: 26 July 2013

\section{References}

1. Musharraf SG, Shoaib M, Najam-ul-Haq M: Validated TLC-densitometry method for the simultaneous analysis of pyrethroid insecticides in agricultural and domestic products. Chem Cent J 2012, 6:93-97.

2. Waghorn GC, McNabb WC: Consequences of plant phenolic compounds for productivity and health of ruminants. P Nutr Soc 2003, 62:383-392.

3. Chankvetadze B, Yamamoto C, Okamoto Y: Enantioseparation of selected chiral sulfoxides using polysaccharide-type chiral stationary phases and polar organic, polar aqueous-organic and normal-phase eluents. J Chromatogr A 2001, 922:127-137.

4. Loukotkova L, Rambouskova ME, Bosakova Z, Tesarova E: Cellulose tris(3,5 dimethylphenylcarbamate)-based chiral stationary phases as effective tools for enantioselective HPLC separation of structurally different disubstituted binaphthyls. Chirality 2008, 20:900-909.

5. Franco $P$, Minguillón C, Oliveros L: Solvent versatility of bonded cellulosederived chiral stationary phases for high-performance liquid chromatography and its consequences in column loadability. J Chromatogr A 1998, 793:239-247.
6. Meidan Y: Chiral drug analysis. Chem Eng News 1990, 68:38.

7. Bae I, Park JH, Choi SH: Synthesis of chiral stationary phase via surfaceinitiated atom transfer radical polymerization of vinylated cellulose 3 , 5-dimethylphenylcarbamate. Polym Int 2011, 60:833-838.

8. Oliveros L, Senso A, Minguillon C: Benzoates of cellulose bonded on silica gel: Chiral discrimination ability as high-performance liquid chromatographic chiral stationary phases. Chirality 1997, 9:145-149.

9. Bai LY, Zhang YP, Deng PH, Zhang YJ, Chen J: Enantioseparation of typical pesticides using cellulose carbamate stationary phases by capillary liquid chromatography. Asian J Chem 2012, 24:4917-4922.

10. Zhang JM, Wu J, Cao Y, Sang SM, Zhang J, He JS: Synthesis of cellulose benzoates under homogeneous conditions in an ionic liquid. Cellulose 2009, 16:299-308.

11. Wilkes JS: A short history of ionic liquids - from molten salts to neoteric solvents. Green Chem 2002, 4:73-80.

12. Ananda S: Amarasekara, Onome S, Owereh: Homogeneous phase synthesis of cellulose carbamate silica hybrid materials using 1-n-butyl3-methylimidazolium chloride ionicliquid medium. Carbohyd Polym 2009, 78:635-638.

13. Swatloski RP, Spear SK, Holbrey JD, Rogers RD: Dissolution of cellose with ionic liquids. J Am Chem Soc 2002, 124:4974-4975.

14. Wu J, Zhang J, Zhang H, He JS, Ren Q, Guo ML: Homogeneous acetylation of cellulose in a new ionic liquid. Biomacromolecules 2004, 5:266-268.

15. Cao Y, Zhang J, He JS, Li HQ, Zhang Y: Homogeneous Acetylation of Cellulose at Relatively High Concentrations in an lonic Liquid. Chinese J Chem Eng 2010, 18:515-522.

16. Okamoto $Y$, Kaida Y: Resolution by high-performance liquid chromatography using polysaccharide carbamates and benzoates as chiral stationary phases. J Chromatogr A 1994, 666:403-419.

17. O'Brien T, Crocker L, Thompson R, Thompson K, Toma PH, Conlon DA, Feibush B, Moeder C, Bicker G, Grinberg N: Mechanistic aspects of chiral discrimination on modified cellulose. Anal Chem 1997, 69:1999-2007.

18. Wang $P$, Jiang SR, Liu DH, Wang P, Zhou ZQ: Direct enantiomeric resolutions of chiral triazole pesticides by high-performance liquid chromatography. J Biochem Biophys Meth 2005, 62:219-230.

doi:10.1186/1752-153X-7-129

Cite this article as: Liu et al:: Green synthesis of a typical chiral stationary phase of cellulose-tris(3,5-dimethylphenylcarbamate). Chemistry Central Journal 2013 7:129.

\section{Publish with ChemistryCentral and every scientist can read your work free of charge \\ "Open access provides opportunities to our colleagues in other parts of the globe, by allowing anyone to view the content free of charge." W. Jeffery Hurst, The Hershey Company. \\ - available free of charge to the entire scientific community \\ - peer reviewed and published immediately upon acceptance \\ - cited in PubMed and archived on PubMed Central \\ - yours - you keep the copyright \\ Submit your manuscript here: \\ http://www.chemistrycentral.com/manuscript/

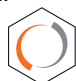 \\ Chemistry Central}

